$$
\text { CONF-9606252--2 }
$$

UCRL-JC-123636

\title{
Positron Beam Lifetime Spectroscopy of Atomic Scale Defect Distributions in Bulk and Microscopic Volumes
}

R.H. Howell

T.E. Cowan

REOEVED

J. Hartley

P. Sterne

Aบ6 161996

OSTI

This paper was prepared for submittal to the

The 7th International Workshop on

Slow-Positron Beam Techniques for Solids and Surfaces

Unterageri, Switzerland

June 2-7, 1996

May 1996
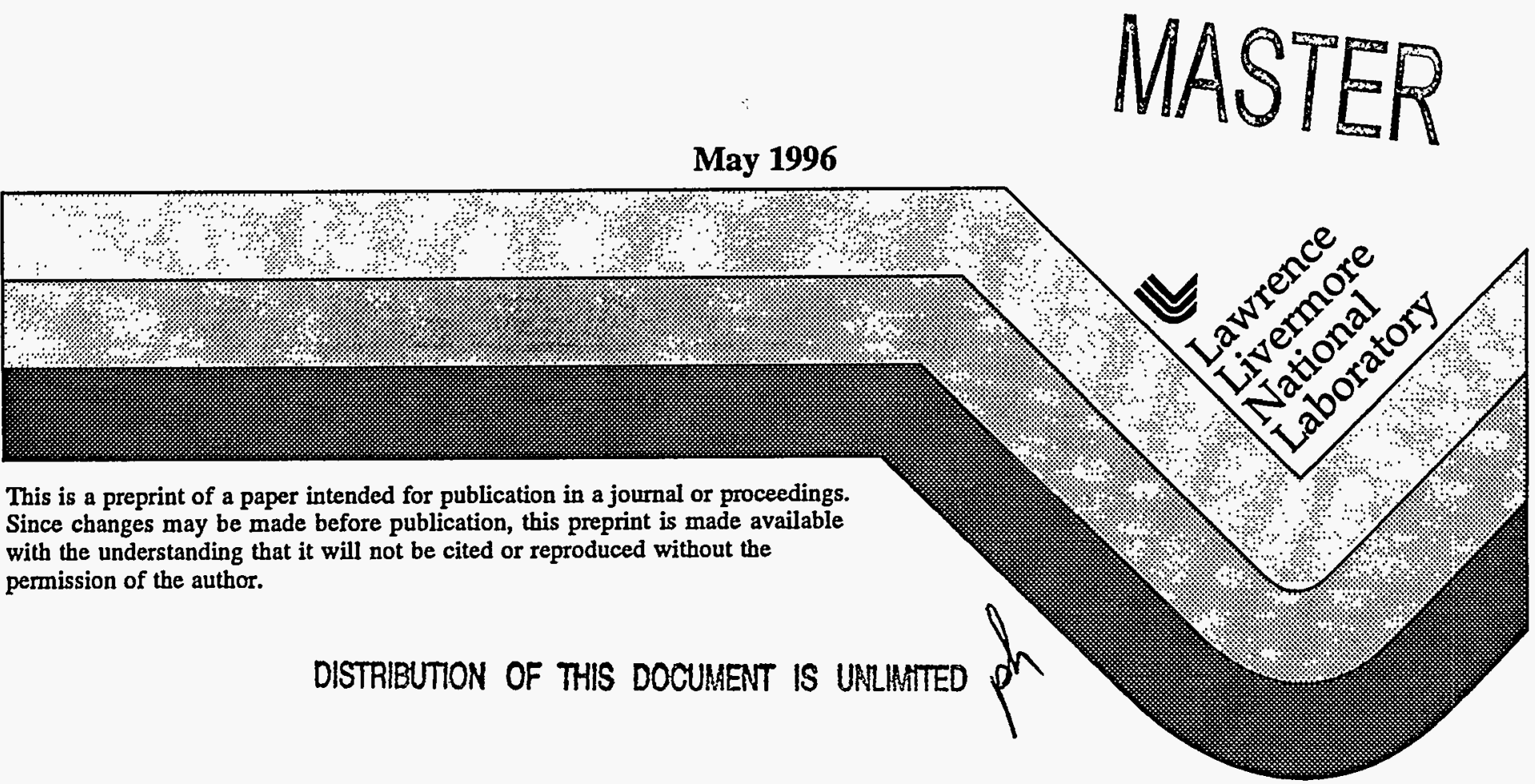


\section{DISCIAIMER}

This document was prepared as an account of work sponsored by an agency of the United States Government. Neither the United States Government nor the University of California nor any of their employees, makes any warranty, express or implied, or assumes any legal liability or responsibility for the accuracy, completeness, or usefulness of any information, apparatus, product, or process disclosed, or represents that its use would not infringe privately owned rights. Reference herein to any specific commercial product, process, or service by trade name, trademark, manufacturer, or otherwise, does not necessarily constitute or imply its endorsement, recommendation, or favoring by the United States Government or the University of California. The views and opinions of authors expressed herein do not necessarily state or reflect those of the United States Government or the University of California, and shall not be used for advertising or product endorsement purposes. 


\section{DISCLAIMER}

Portions of this document may be illegible in electronic image products. Images are produced from the best available original document. 
Positron Beam Lifetime Spectroscopy of Atomic Scale Defect Distributions in Bulk and Microscopic Volumes

Richard H. Howell, Thomas E. Cowan, Jay Hartley, and Philip Sterne

Lawrence Livermore National Laboratory, Livermore CA 94550

Ben Brown

Mount Holyolk College Hadley, MA 01033,

We are developing a defect analysis capability based on two positron beam lifetime spectrometers: the first is based on a $3 \mathrm{MeV}$ electrostatic accelerator and the second on our high current linac beam. The high energy beam lifetime spectrometer is operational and positron lifetime analysis is performed with a $3 \mathrm{MeV}$ positron beam on thick sample specimens. It is being used for bulk sample analysis and analysis of samples encapsulated in controlled environments for in situ measurements. A second, low energy, microscopically focused, pulsed positron beam for defect analysis by positron lifetime spectroscopies under development at the LLNL high current positron source. This beam will enable defect specific, 3-dimensional maps of defect concentration with sub-micron location resolution and when coupled with first principles calculations of defect specific positron lifetimes it will enable new levels of defect concentration mapping and defect identification.

\section{Background}

There is a general need in the materials science and positron research community and in the semiconductor and polymer industries to understand the effects of atomic scale defects resulting from fatigue, processing, irradiation or other causes. Macroscopic properties such as work hardening, radiation damage, electromigration induced failure of current carrying lines and aging of mechanical properties of plastics are determined by the production and migration of vacancy related defects. Current needs require knowledge of both the defect identity and concentration averaged over small distances for many kinds of samples including thin films, small crystals, buried interfaces, small features and the near-surface regions of many materials. Such knowledge is required for the broad range of problems including electromigration in electronic interconnects in the semiconductor industry, polymer properties related to hole volume in the polymer industry and validation of calculations in quantum mechanical and microscopic materials models in basic solid state research.

\section{Positron Annihilation Lifetime Spectroscopy}

There are many probes for interstitial defects including several that produce depth dependent data. Probing vacancy type defects is more challenging and positron spectroscopy is unique in its ability to spatially analyze dilute quantities of buried, atomic scale vacancy defects and determine their location in three 
dimensions. This ability derives from special properties of the positron. When positrons are introduced into a solid material they quickly slow to thermal energies and after a short period of diffusion annihilate with an electron. Positron spectroscopies are based on techniques for detecting properties of annihilation gamma-rays to determine properties of the participating electron.

The annihilation site of the positron determines which electron distribution will be sampled by the positron. Positrons in defect free material span a large volume and sample all of the available electrons. However in material containing open volume defects the positron will strongly interact and trap at the defect site. In this case the electron population available for annihilation is confined to the defect volume and leads to distinct changes in positron lifetimes. Consequently positron lifetime spectroscopy is a unique, non-destructive tool for the detection of atomic scale defects.

As a defect spectroscopy, positron lifetime spectroscopy can provide the defect size, concentration and location for open volume defects in most solid materials. The correlation between low local electron density and larger size defects leads to longer positron lifetimes for larger defects. Using the lifetime results from positron annihilation lifetime spectroscopy and lifetime values obtained from theoretical calculations we can estimate the size and local environment of vacancies, vacancies at dislocations, and voids, and thereby identify unknown defect species in metals, semiconductors and insulators. Concentration values are determined from the fraction of positrons that trap at a defect before annihilating. The rate for trapping in defects is high even for single atomic vacancies and so positron spectroscopies are sensitive to even low levels of defect concentrations, $10^{-3}$ to $10^{-6}$. Positron annihilation lifetimes can be measured by the difference between the time of positron entry into the sample and the later time of annihilation. A typical lifetime spectrum would contain the distribution of $10^{6}$ individual positron time histories, see figure $1 \mathrm{a}$.

Since the positron provides spectroscopic measurements containing both concentration and defect size information, defect location depends solely on where the positron is implanted in the subject material. The end of range of an implanted positron is determined by the positron energy, implantation location and statistical variations in the slowing down process, such as illustrated in figure $1 \mathrm{~b}$. Thus a highly focused, low energy beam can be used to determine the size and concentration of a defect population over an extremely small volume. High energy positrons sample a much larger volume since they are very penetrating and broadly distributed by statistical variations of the energy loss. 

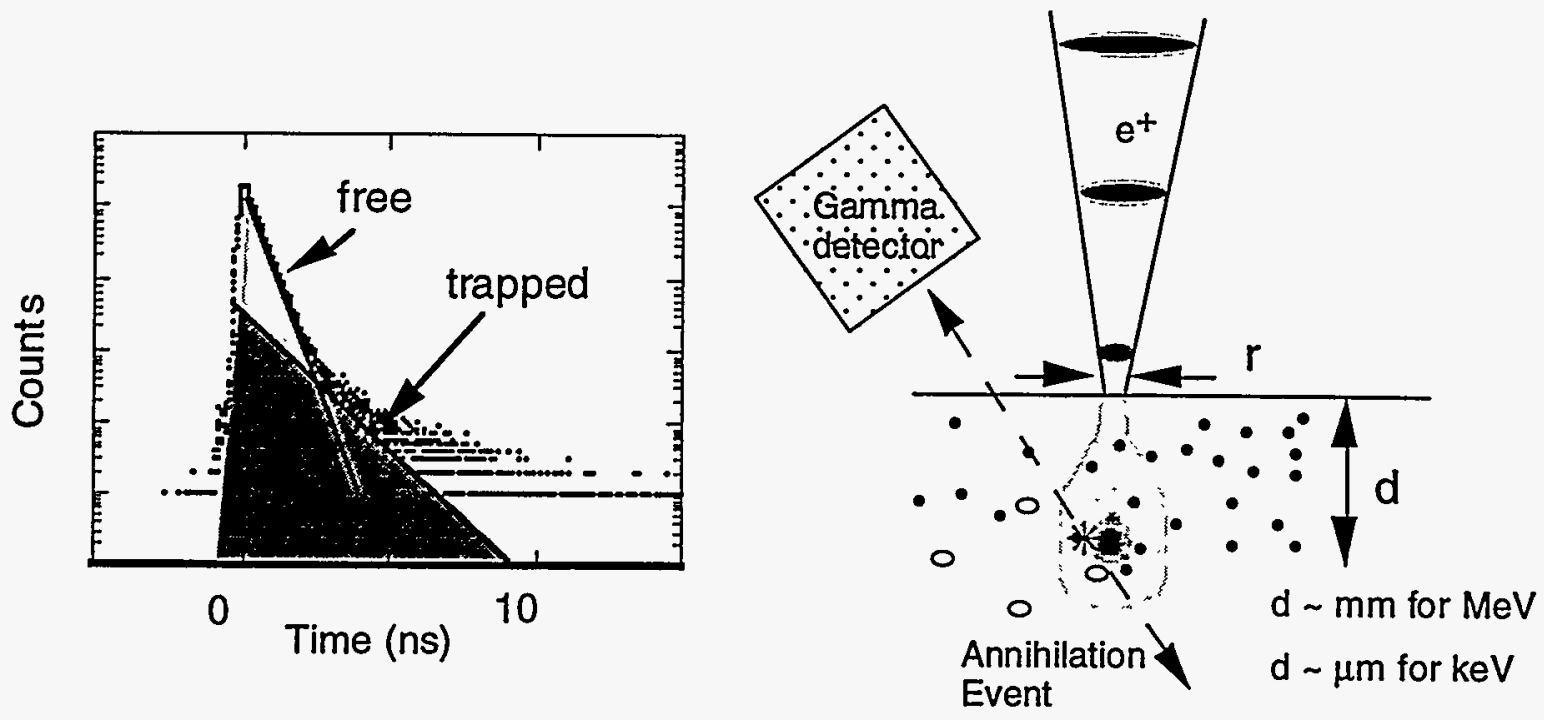

Figure 1. In a positron annihilation lifetime spectrum defect size is determined by the annihilation rates in the defect and defect concentration by the ratio of defect-trapped to total annihilation events. Positrons are highly effective at trapping at defects and are sensitive to low concentrations of defects. Defect location is determined by defining the end point of the implanted positron by controlling positron beam size and energy to confine the positrons to a volume of scale $\sim r^{2} d$.

\section{Other defect analytical techniques}

There are other methods for the general detection of open volume defects. Some of the best known are microscopies such as transmission electron microscopy, atomic force microscopy, scanning tunneling microscopy, and optical microscopy. There are also other defect spectroscopies such as small angle $x$-ray or neutron scattering. Each of these techniques have specific regions of high sensitivity and resolution. The capabilities of these techniques and positron annihilation lifetime spectroscopy are compared in figure 2. In figure 2-a each method is displayed 'by its ability to resolve or detect defects of some size at some sample depth. We see that positron annihilation lifetime spectroscopy is effective in providing size information at any sample depth for defect sizes below the effective resolution of other generally applied techniques. Figure 2-b shows the same comparison for defect concentration. Since defect concentrations are determined by the probability of a positron trapping during thermal diffusion, concentration values can also be determined with similar accuracy for any sample depth. 

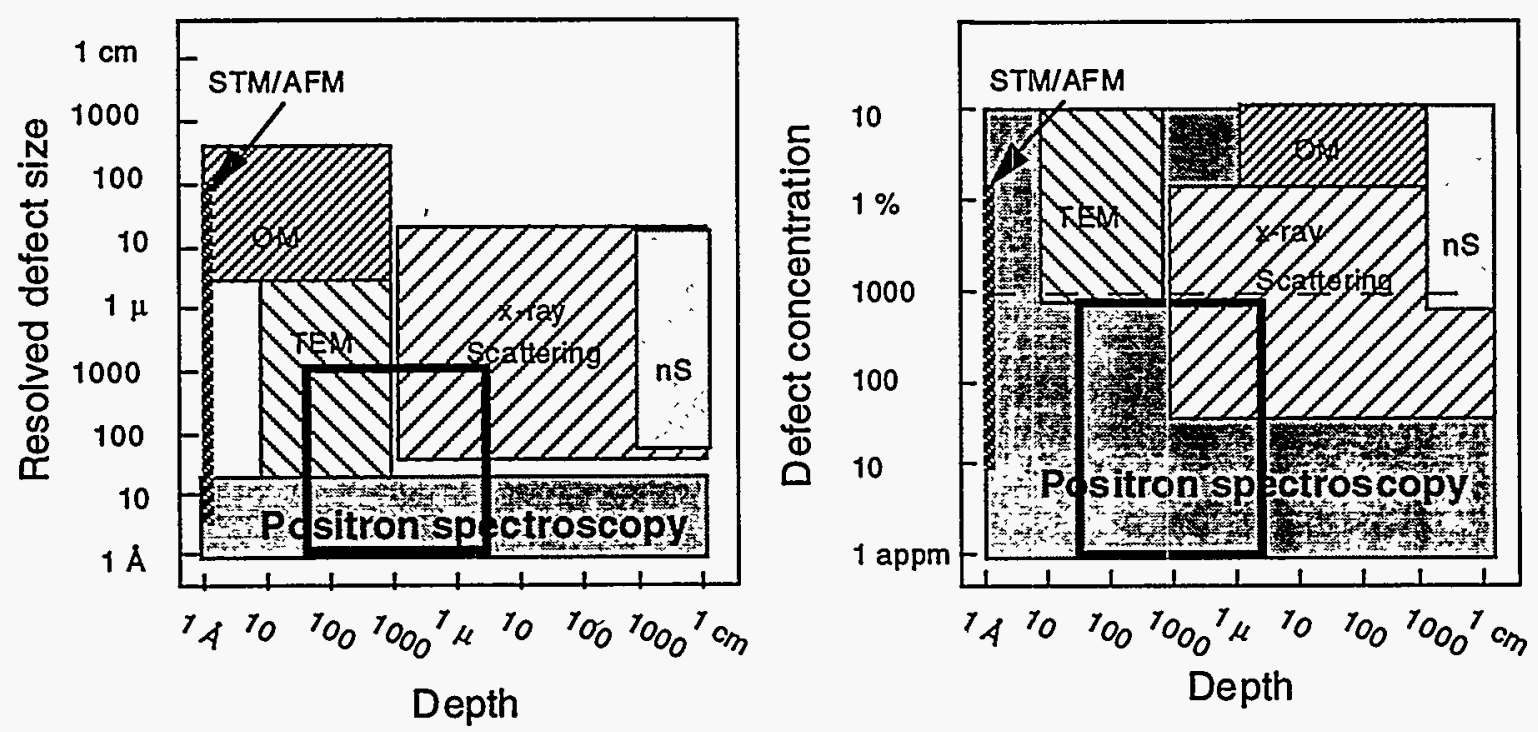

Fine line interconnects

Figure 2. Positron annihilation lifetime analysis fills a special niche in the group of techniques for general vacancy defect analysis, optical microscopy, OM, neutron scattering, ns, transmission electron microscopy, TEM, scanning tunneling microscopy, STM and atomic force microscopy, AFM, and x-ray scattering. Positron techniques are both highly sensitive and can resolve the size of atomic vacancies at any depth in a sample. The solid black line outlines the range of interest for studies of fine lines used as electronic interconnects.

\section{Bulk Average Positron Spectroscopic Analysis}

We have obtained higher throughput and a significant easing of sample geometry restrictions over conventional radioactive source techniques by performing positron annihilation lifetime analysis analyses with a positron beam accelerated in our $3 \mathrm{MV}$ Pelletron electrostatic accelerator. This system is modeled on one in use at Stuttgart, Germany[ 1 ]. Lifetime measurements are performed by detecting the time difference between the time of passage of the positron through a $100 \%$ efficient detector outside the sample and the annihilation gamma ray after implantation. This results in single sample measurements in a relaxed geometry with significantly higher counting rates and suppressed backgrounds in the positron annihilation lifetime spectra.

The accelerator-detector configuration is shown in figure 3. Positrons are captured from a $100 \mathrm{mCi}$ source of ${ }^{22} \mathrm{Na}$ and 2 micron $\mathrm{W}$ moderator foil placed in the terminal of a 3 MV Pelletron electrostatic accelerator. Both fast and thermalized positrons are focused by electrostatic lenses in the terminal and a magnetic lens after acceleration. The positron beam is focused on to a $3 \mathrm{~mm}$ thick plastic detector that times the implantation of each positron. The positrons are then implanted into the sample and annihilation gamma rays are detected by a $\mathrm{BaF}_{2}$ annihilation gamma-ray detector. Positrons not annihilating in the sample are rejected by a plastic scintillator anti-coincidence detector. The initial positron beam contains 6 $10^{5} \mathrm{e}^{+} / \mathrm{s}$ and final counting rates are $\sim 1000 \mathrm{c} / \mathrm{s}$ in a close geometry. 
At these rates bulk average defect analysis in engineering samples of encased materials can be performed on up to 50 samples a day. Energetic $3 \mathrm{MeV}$ positrons will implant to $\mathrm{mm}$ depths in most materials and can penetrate thin windows to reach the sample. Thus measurements can be performed on material encased in vacuum chambers or other necessary containment. With our mm beam spot, defect analysis with this instrument provides bulk values averaged over $\sim 0.1 \mathrm{~cm}^{3}$ sample volume. The beam-detector geometry also allows coincident measurement of both electron momentum and electron density by separate positron spectroscopic techniques. This provides identification of gas filling in voids and a more detailed description of the local structure surrounding a vacancy defect.

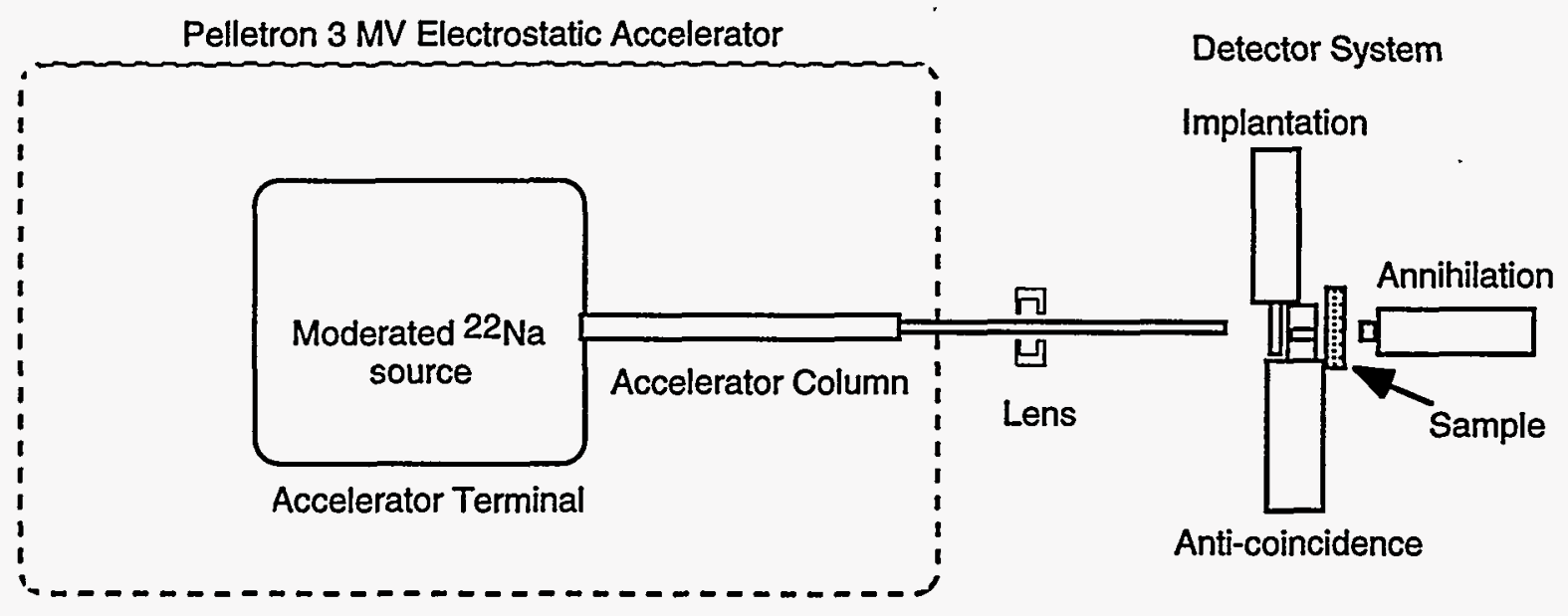

Figure 3. Schematic diagram of the critical components of the high energy beam positron annihilation lifetime system.

\section{Technical capabilities of a pulsed, positron microprobe}

We have begun to design, construct and test an intense, pulsed, sub-micron size positron beam for high spatial resolution positron annihilation lifetime spectroscopy. The instrument is designed to run for either spot analysis or in scanning mode to provide a three dimensional map of defect concentrations. By coupling our apparatus to the highest current positron beam in existence $\left(10^{10} \mathrm{e}^{+\mathrm{s}^{-}}\right.$ 1) we will provide an intense, variable energy ( 0 to $50 \mathrm{keV})$, pulsed (less than $100 \mathrm{ps}$ ), high intensity ( over $10^{7} \mathrm{e}^{+} \mathrm{s}^{-1}$ ) sub-micron size positron beam. Our intense beam and positron microprobe enable us to maintain high data rates while detecting and identifying the depth dependent concentration of vacancies, voids, gas filled voids and other negatively charged defects to a depth of a few microns and at typical depth and lateral resolutions of less than 0.1 micron. 
To build the microprobe we are integrating the concepts of existing designs for pulsed positron beams [ 2,3 ], focused positron beams [ 4 ] and pulsed microprobes [ 5 ] into an optimized design to specifically utilize the physical layout, high beam intensity and timing characteristics of the linac positron beam. A schematic diagram of the key elements of a pulsed positron microprobe system can be seen in figure 3. The pulsed microprobe instrument is physically and logically separated into two stages: a primary stage containing the high current positron beam, a Penning trap beam stretcher, the pulsing system and initial focusing, and a secondary stage containing the final beam acceleration and microscopic focusing.

The first stage has been designed to take advantage of the unique characteristics of our linac source. Ultimate operation of a pulsed system is limited by the energy distribution of the initial source. By taking advantage of the characteristics we will use the Penning trap stretcher to invert the time and energy distribution of our high current positron beam, $\Delta \mathrm{E} \sim 4 \mathrm{eV}, \Delta \mathrm{t} \sim 3 \mathrm{~ms}$, resulting in a nearly $\mathrm{DC}$ beam with an energy distribution, $\Delta \mathrm{E} \sim 20 \mathrm{meV}[3]$. This is significantly more monoenergetic than a cooled moderator. The narrow energy spread of our beam as it enters the pulsing system will reduce the time of flight dispersion from that source and will allow us to operate at lower transport voltages and obtain short duration pulses. - The required power in all subsequent pulsing electronics is correspondingly lower and we can effectively use a continuous train of pulses from an arbitrary wave form generator shaped to the ideal bunching profile to achieve a high efficiency and sub-nanosecond time bunching in the first stage of the buncher. After exiting the prebuncher, the beam will be accelerated, extracted from the magnetic transport system, deaccelerated, and focused onto a tungsten remoderator by a single pole magnetic lens [ 6 ]. Final bunching and chopping will be done on the reemitted beam using conventional designs and sine wave pulse shapes. The beam will then be focused onto a second remoderator at the entrance of the second stage.

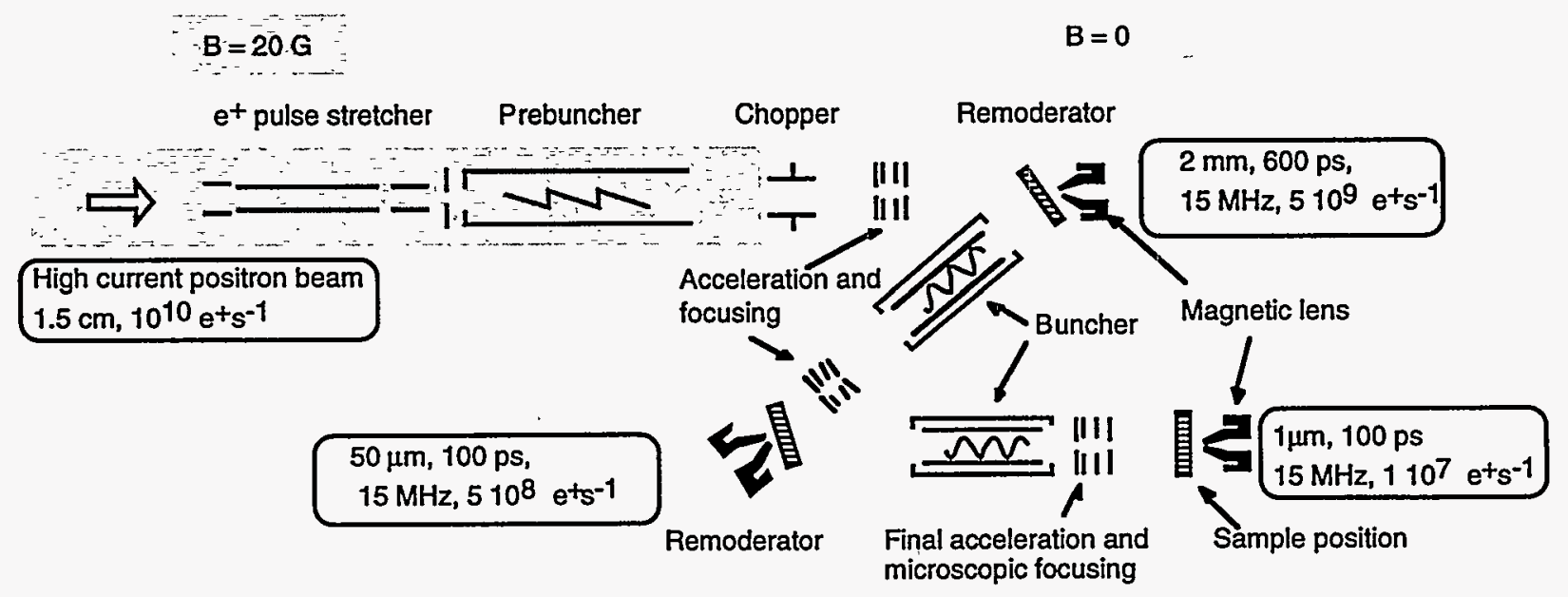


Figure 3 Schematic of the main elements of the 3-D pulsed positron microbeam system. The final beam of more than $10^{7} \mathrm{e}^{+} \mathrm{s}^{-1}$ will be less than 1 micron diameter and 0.5 to $50 \mathrm{keV}$. Scanning in the plane of the sample surface will be done by sweeping the beam for small areas and positioning the sample for large areas. Detailed design of the pulsing and focusing systems began on December 1, 1995

We are currently installing the elements of the first stage. The second stage will follow published designs[ 7 ]. Details of the sample chamber, detector systems and other associated components will be finalized in 1996. Scanning over small areas will be accomplished by beam steering (not shown in figure 3). Larger areas will be scanned by moving the sample using micropositioning of the sample stage.

\section{Applications of the 3-D microprobe and positron related defect analysis}

The role of defects in thin films and at interfaces is central to understanding the properties of many advanced materials of programmatic and commercial interest. For example the role of defects in the nucleation of voids in electromigration and stress voiding is pivotal in the development of smaller and better electronic device structures, the molecular free volume elements in polymeric coatings is a determining factor in their mechanical and aging properties, and the defect characteristics of metals and alloys determines their strength and corrosion resistance.

Defects are a generic problem that affect much of materials behavior. The evolution of defects during conditions of unusual temperature and pressure or over long periods of time determines the ultimate strength and corrosion of many systems. Consequently a broad range of problem ranging from identifying the defect species in high temperature superconductors or the defect active in the oxygen transport in uranium corrosion to the validation of bonds between metals require defect identification and quantification. Many of these problems are well suited to positron lifetime analysis which is a non-destructive technique with specific defect response.

Electrical properties of semiconducting materials and devices are determined by defects that have intentionally been introduced or occur accidentally. There is an ongoing need for non-destructive analysis of defects for both research and production line quality assurance. Positron annihilation analysis with a beam currently provides the one dimensional depth distribution of defects in semiconducting materials and structures. Measurement of the annihilation characteristics of mono-energetic positrons implanted at known depths can be related to the charge carrier diffusion, the interfacial defect concentrations, and the defect population of thin layered materials. Measurements with large spot beams have already provided unique data on systems such as ion implanted silicon, silicon epitaxially grown on silicon, silicon implanted by oxygen, and stress voiding and electromigration. Semiconductor interface analysis dominated the discussions of 
positron beam applications at the last topical meeting, SLOPOS6, held May 1994, in Japan.

The 3-D pulsed positron microprobe can provide new answers in semiconductor device problem areas. Some areas where our experiments with the microprobe can have an early impact were discussed at the first users workshop held in Livermore in June 1995. They are electromigration and stress voiding in interconnects, defects produced during processing that affect interface quality in gate oxides in CMOS junctions, defects associated with ion bombardment, and flaws and other features in the polymeric packaging.

Macroscopic and electrical properties of polymers can be understood using theories based on the free volume of the material. Application of the theory, which unifies the discussion of impact strength, elastic properties, some electrical properties gas permeability and aging has been limited by the lack of definitive measurements of the free volume characteristics in real materials. Positron annihilation analysis is capable of measuring the free volume fraction and microscopic size distribution in a non-destructive setting.

Plastics have a wide range of applications and understanding their characteristics during fabrication and use is of major interest to nearly every plastics application. In some cases bulk volume distributions of the hole volume is sufficient. However much of plastic use is in thin coatings and irregular shaped objects. Using positron annihilation spectroscopic measurements in the microprobe we will provide microscopic determinations of properties that determine behavior in aging, creep fatigue and processing induced stress in thin films and at specific stress points.

We wish to thank Drs. W. Triftshäuser, G. Kögel, P. Speer, and D.T. Suzuki for their valuable conversations on beam pulsing and focusing. This work was performed under the auspices of the U.S. Department of Energy by Lawrence Livermore National Laboratory under contract No. W-7405-ENG-48.

1. W. Bauer, J. Briggmann, H.-D. Carstanjen, S. H. connell, W. Decker, J. Diehl, K. Maier, J. Major, H. E. Schaefer, A. Seeger, H. Stoll and E. Widmann, Nucl. Inst. and Meth. B50 , 300 (1990)

2. P. Willutzki, J. Störmer, G. Kögel, P.Speer, D. T. Britton, R. Steindl and W. Triftshäuser, Materials Science Forum 175-178, 237 (1995)

3. R. Suzuki, T. Mikado, M. Chigaki, H. Ohgaki, and T. Yamazaki Applied Surface Science $\underline{85}, 87$ (1995) 
4. G.R. Brandes, K. F. Canter, T. N. Horsky and P.H. Lippel and A. P. Mills, Rev. Sci. Instrum. $\underline{59} 228$ (1988)

5. A. Zecca, R. S. Brusa, M. P. Duarte-Naia, G. PP Karwasz, J. Paridaens, A. Piazza, G. Kögel, P. Sperr, D. T. Britton, K. Uhlmann, P. Willutzki and W. Triftshäuser, Europhys. Lett. 29,617 (1995)

6. K. Uhlman, D. T. Britton and G. Kögel, Optik $\underline{98} 5$ (1994)

7. K. Uhlmann, D. T. Britton and G. Kögel, Meas. Sci. Technol. 6932 (1995) 